\title{
ANTIMICROBIAL ACTIVITY OF LACTOBACILLUS PLANTARUM AGAINST PATHOGENIC AND FOOD SPOILAGE MICROORGANISMS: A REVIEW
}

\author{
T. DINEV ${ }^{1}$, G. BEEV ${ }^{1}$, M. TZANOVA ${ }^{1}$, S. DENEV ${ }^{1}$, D. DERMENDZHIEVA ${ }^{2}$ \\ \& A. STOYANOVA ${ }^{3}$ \\ ${ }^{1}$ Department of Biochemistry, Microbiology and Physics; ${ }^{2}$ Department of \\ Applied Ecology and Animal Hygiene; ${ }^{3}$ Department of Plant Production; \\ Faculty of Agriculture, Trakia University, Stara Zagora, Bulgaria
}

\section{Summary}

Dinev, T., G. Beev, M. Tzanova, S. Denev, D. Dermendzhieva \& A. Stoyanova, 2018. Antimicrobial activity of Lactobacillus plantarum against pathogenic and food spoilage microorganisms: A review. Bulg. J. Vet. Med., 21, No 3, 253-268.

One of the most important properties of probiotic bacteria is their antimicrobial activity against many species of microorganisms which could be useful to prevent food spoilage caused by certain sensitive bacteria and fungi as well as to control the speed of propagation of potentially pathogenic bacteria by probiotic application. Lactobacillus plantarum is considered one of the probiotic bacteria with broadest spectrum of antibacterial activity which makes it useful in veterinary, human medicine and food industry. According to a number of studies Lactobacillus plantarum exerts inhibitory activity against many Gram-positive and Gram-negative bacteria - Escherichia coli (including E. coli 0157:H7), Pseudomonas aeruginosa, Helicobacter pylori, Yersinia enterocolitica, Campylobacter jejuni, Listeria monocytogenes, Staphylococcus aureus, Klebsiella, Salmonella, Shigella, Bacillus, Clostridium, Enterococcus, Lactobacillus spp., etc. as well as a number of moulds and yeasts - Aspergillus, Fusarium, Mucor, Candida spp., etc. The main antibacterial compounds of Lactobacillus plantarum are the bacteriocins and organic acids whereas the antifungal compounds are the organic acids, hydroxy fatty acids and cyclic dipeptides. Because of the high antifungal activity of some L. plantarum strains against food spoilage microorganisms they can be used as effective biopreservatives in food industry. Also, some $L$. plantarum strains could be applied as supporting therapeutic agents in treatment of infections caused by the corresponding susceptible microorganisms.

Key words: antimicrobial activity, Lactobacillus plantarum, food spoilage microorganisms, pathogens

\section{INTRODUCTION}

Spoilage of food products by bacteria and fungi is a worldwide problem. They can cause extensive damage of the food such as unpleasant smell, taste or appearance as well as formation of harmful substances for the consumer's health. Also, another 
important aspect of food contamination by microorganisms is the presence of potentially pathogenic species, which pose a great risk for the human and animal health (Broberg et al., 2007). One of the most important properties of probiotic bacteria is their antimicrobial activity against many species of microorganisms which could be useful in order to avoid the constant application of antibiotics and to control the speed of propagation of potentially pathogenic intestinal bacteria (Arias et al., 2013).

Lactobacillus plantarum (L. plantarum) is a member of lactic acid bacteria (LAB). It is an important species taking part in the fermentation of many plant products (silage, sauerkraut, brined olives, pickles), sourdough, cheeses, fermented sausages and stockfish (Cebeci \& Gürakan, 2003). It belongs to the probiotic Lactobacillus species inhabiting the human digestive system and producing bacteriocins, exopolysaccharides, extracellular proteins and lipoteichoic acids. They improve the health and physiology of the host by interacting with epithelial cells and enhancing the host immune system (Arasu et al., 2016). Recently L. plantarum has been applied in human medicine for treatment of chronic inflammation associated with various diseases including cancer, Parkinson's disease, Alzheimer's disease and cardiovascular diseases (Woo et al., 2014). Kurhan \& Çakir (2016) reported that L. plantarum has DNA-bioprotective effect reducing the aflatoxin $\mathrm{B}_{1}$ genotoxic effect on colon adenocarcinoma (Caco-2) cells. Comparative studies between different probiotics showed that L. plantarum strains demonstrated the broadest spectrum of antimicrobial activity among the probiotic bacteria examined (Dembélé et al., 1998; Wang et al., 2010; Ren et al., 2014; Dubourg et al., 2015; Davoodabadi et al., 2015).
Considering the increasing importance of the LAB as antibiotics alternative, the knowledge of the antimicrobial activity of the main LAB species and L. plantarum in particular is of especially high significance. The antimicrobial activity of $L$. plantarum can show if its products can be helpful in the treatment of a particular infection or if they can prevent the development of undesirable microbiota in food products. In that way the supplementation of $L$. plantarum products could be made on the basis of the antimicrobial activity of the particular L. plantarum strain.

\section{ANTIMICROBIAL COMPOUNDS PRODUCED BY L. PLANTARUM}

\section{Bacteriocins}

Bacteriocins are ribosomally synthesised antimicrobial peptides produced by various bacteria, including LAB. Some of them have great potential in food preservation and can reduce or eliminate the need for addition of chemical preservatives or the intensity of processing the food and in that way can satisfy the demand for high-quality foods (Perez et al., 2014). To guarantee bacteriocin effectiveness when supplemented to the food it should be tested against specific target microorganisms in the type of food for which they are intended to be used. Most of the bacteriocins kill the susceptible bacteria by inducing permeabilisation and pore formation on the cytoplasmic membrane or by interactions with essential enzymes (Wen et al., 2016). Because bacteriocins are degraded by the proteolytic enzymes of the gastrointestinal tract and seem to be non-toxic and non-antigenic to animals and humans they can be used to improve the safety and shelf-life of many food products (Amenu, 2013). When selecting bacteriocins for food application 
the following criteria should be considered: the bacteriocin-producing strain should be generally recognised as safe; the bacteriocin should: 1) have a broad spectrum of inhibition against a variety of food-borne pathogens or specificity against a particular pathogen specific for given food; 2) have high degree of heat stability; 3) lead to beneficial effects in the product such as improved safety and quality; 4) have a high specific activity (O'Sullivan et al., 2002).

\section{Organic acids}

L. plantarum is facultative heterofermentative species that ferment carbohydrates to produce lactic acid and ethanol or acetic acid. Organic acid lowers the local $\mathrm{pH}$ and therefore inhibits the growth of bacteria, sensitive to acidic conditions. The low $\mathrm{pH}$ makes organic acids liposoluble, allowing them to break through the cell membrane and reach the cytoplasm of target microorganisms (Haller et al., 2001). However, the microorganisms differ considerably in their sensitivity to lactic acid. At pH 5.0 lactic acid exert inhibitory activity towards spore- forming bacteria but is ineffective against yeast and moulds (Amenu, 2013). Acetic and propionic acids produced by $L$. plantarum strains through heterofermentative pathways, may interact with cell membranes and cause intracellular acidification and protein denaturation (Urga et al., 1992). They have higher antimicrobial activity than lactic acid due to their higher $\mathrm{pKa}$ values (lactic acid 3.08, acetic acid 4.75, and propionic acid 4.87), and higher percent of undissociated acids than lactic acid at a given $\mathrm{pH}$ (Earnshaw, 1992).

\section{Hydroxy fatty acids}

There are several studies indicating that 3hydroxy fatty acids have antifungal activity which is due to detergent-like properties of the compounds that alter cellular membrane structure in the target organisms (Sjögren et al., 2003). According to studies on LABproduced antimicrobial fatty acids, they have a broad spectrum of antifungal activity (Sjögren et al., 2003; Dalié et al., 2010).

\section{Hydrogen peroxide}

Hydrogen peroxide is produced by $L$. plantarum and the other LAB in the presence of oxygen as a result of the action of flavoprotein oxidases or NADH peroxidase. The antimicrobial activity of hydrogen peroxide could be the result of the oxidation of sulfhydryl groups causing denaturation of a number of enzymes, and from the peroxidation of membrane lipids leading to increased membrane permeability (Amenu, 2013). Hydrogen peroxide can be a precursor for the production of bactericidal free radicals such as superoxide $\left(\mathrm{O}_{2}^{-}\right)$and hydroxyl $\left(\mathrm{OH}^{-}\right)$radicals which can damage DNA (Byczkowski \& Gessner, 1988). According to Gerez et al. (2013) hydrogen peroxide does not exert any antifungal activity.

\section{Carbon dioxide}

Carbon dioxide is mainly produced by heterofermentative LAB. Because L. plantarum is facultative heterofermentative species, depending on the carbon source it can switch between using heterofermentative and homofermentative ways of metabolism (Kleerebezem et al., 2003). The precise mechanism of the antimicrobial action of carbon dioxide is still poorly understood. However, carbon dioxide may play a role in creating an anaerobic environment, which inhibits enzymatic decarboxylations, and the accumulation of carbon dioxide in the membrane lipid bilayer is a possible cause for permeability dysfunction (Eklund, 1984). 


\section{ANTIMICROBIAL ACTIVITY OF \\ L. PLANTARUM AGAINST \\ PATHOGENIC AND FOOD \\ SPOILAGE MICROORGANISMS}

\section{Antibacterial activity of L. plantarum}

According to the experimental data $L$. plantarum is active against many Gramnegative pathogens and food spoilage microorganisms - Escherichia coli (including enteropathogenic, enterotoxigaenic, enteroinvasive, multidrug-resistant enteroaggregative E. coli and E. coli 0157:H7), Pseudomonas aeruginosa, Yersinia enterocolitica, Campylobacter jejuni, Helicobacter pylori, Klebsiella, Salmonella, Shigella spp., etc. (Table 1). Also L. plantarum exerts inhibitory activity against a variety of potentially harmful Gram-positive bacteria - Listeria monocytogenes, Staphylococcus aureus and some members of the genera Bacillus, Clostridium, Enterococcus, Lactobacillus, etc. (Table 2). It is important to emphasise that the antimicrobial activity of $L$. plantarum (and the other LAB) is strain specific, i.e. only some strains of $L$. plantarum are inhibitory toward specific strains of the microbial species (Denev et al., 2015). There are three mechanisms that could explain the antimicrobial activity of LAB and L. plantarum in particular: the production of bacteriocins; the yield of organic acids and other inhibitory substances such as ethanol, carbon dioxide and hydrogen peroxide; and the competition for nutrients (Magnusson et al., 2003).

L. plantarum strains produce a broad range of bacteriocins such as ST28MS, ST26MS, bacST202Ch, bacST216Ch, ST71KS, AMA-K, plantaricin B, D, G, K, K25, S, BN, UG1, S, T, C19, CTC 305, CTC 306, 35d, Q7, MG, 163, ASM1, EF, $\mathrm{JK}, \mathrm{N}, \mathrm{NC} 8, \mathrm{ZJ} 008$, etc. (Enan et al., 1996; Todorov \& Dicks, 2005; Todorov et al., 2007; 2010; Hata et al., 2010; Gong et al., 2010; Martinez et al., 2013; Buntin \& Hongpattarakere, 2014; Zhu et al., 2014; Jiang et al., 2016; Liu et al., 2016; Wen et al., 2016). Different L. plantarum bacteriocins have bactericidal mode of action and diverse antimicrobial spectrum of activity. They usually have narrow spectrum of activity against closely related Gram-positive bacteria from Lactobacillaceae, whereas producer cells are immune to their own bacteriocins (AboAmer, 2013). It is well established that Gram-negative bacteria are intrinsically resistant to bacteriocins produced by $\mathrm{LAB}$ due to the presence of external membrane, which constitutes a physical barrier to the passage and binding of bacteriocins (Pehrson et al., 2015). However, it has been reported that the destabilisation of the outer membrane can make Gram-negative bacteria susceptible to these bacteriocins. It is found that lactic acid acts as a permeabiliser of the outer membrane of Gramnegative bacteria, thus increasing their susceptibility to antimicrobials (including bacteriocins), allowing their molecules to penetrate the bacteria (Alakomi et al., 2000).

There are also some bacteriocins having a broad range of inhibition against Gram-positive and Gram-negative bacteria including food-borne pathogens such as Listeria monocytogenes, Escherichia coli, Staphylococcus aureus, Clostridium perfringens, Pseudomonas spp. etc., and antimicrobial resistance is not likely to be induced after their application (Gong et al., 2010; Todorov et al., 2010). Because the bacteriocins produced by certain strains of L. plantarum sometimes exert antibacterial activity against a large number of Gram-positive and Gram-negative spoilage and pathogenic bacteria, the respective L. plantarum strains are suitable 
Table 1. Antibacterial activity of Lactobacillus plantarum strains against Gram-negative bacteria

\begin{tabular}{|c|c|}
\hline $\begin{array}{l}\text { Spectrum of } L . \text { plantarum } \\
\text { activity }\end{array}$ & References \\
\hline Acinetobacter baumannii & Todorov \& Dicks, 2005 \\
\hline Bacteroides thetaiotaomicron & Dubourg et al., 2015 \\
\hline Campylobacter jejuni & Patel et al., 2013 \\
\hline Citrobacter freundii & Dal Bello et al., 2007 \\
\hline Enterobacter aerogenes & Tambekar \& Bhutada, 2009 \\
\hline Enterobacter cloacae & Dubourg et al., 2015 \\
\hline Erwinia persicina & Jiang et al., 2016 \\
\hline Escherichia coli & $\begin{array}{l}\text { Todorov \& Dicks, 2005; Dal Bello et al., 2007; Todorov et } \\
\text { al., 2007; Tambekar \& Bhutada, 2009; Gong et al., 2010; } \\
\text { Wang et al., 2010; Todorov et al., 2010; Patel et al., 2013; } \\
\text { Buntin \& Hongpattarakere, 2014; Peres et al., 2014; Ren et } \\
\text { al., 2014; Zhu et al., 2014; Davoodabadi et al., 2015; Dub- } \\
\text { ourg et al., 2015; Pehrson et al., 2015; Venkadesan \& Su- } \\
\text { mathi, 2015; Liu et al., 2016; Aoudia et al., 2016; Kumar et } \\
\text { al., 2016; Wen et al., 2016 }\end{array}$ \\
\hline Helicobacter pylori & Sunanliganon et al., 2012 \\
\hline Klebsiella pneumoniae & $\begin{array}{l}\text { Todorov et al., 2007; Tambekar \& Bhutada, 2009; Todorov } \\
\text { et al., 2010; Omemu \& Faniran, 2011; Ren et al., 2014; Khan } \\
\text { \& Kang, } 2016\end{array}$ \\
\hline Pseudomonas spp. & Todorov et al., 2010 \\
\hline P. aeruginosa & $\begin{array}{l}\text { Todorov \& Dicks, 2005; Rodríguez et al., 2012; Peres et al., } \\
\text { 2014; Todorov et al., 2014; Zhu et al., 2014; Dubourg et al., } \\
\text { 2015; Khan \& Kang, 2016; Liu et al., 2016; Wen et al., } 2016\end{array}$ \\
\hline P. fluorescens & Gong et al., 2010 \\
\hline Proteus vulgaris & Dal Bello et al., 2007; Tambekar \& Bhutada, 2009 \\
\hline P. mirabilis & Omemu \& Faniran, 2011; Dubourg et al., 2015 \\
\hline $\begin{array}{l}\text { Salmonella enterica subsp. ari- } \\
\text { zonae }\end{array}$ & Jiang et al., 2016 \\
\hline S. enterica subsp. enterica & Wang et al., 2010; Rodríguez et al., 2012; Jiang et al., 2016 \\
\hline $\begin{array}{l}\text { S. enterica subsp. enterica ser. } \\
\text { Enteritidis }\end{array}$ & $\begin{array}{l}\text { Davoodabadi et al., 2015; Pehrson et al., 2015; Aoudia et al., } \\
2016\end{array}$ \\
\hline$S$. Paratyphi & Buntin \& Hongpattarakere, 2014; Jiang et al., 2016 \\
\hline$S$. Typhi & Tambekar \& Bhutada, 2009; Venkadesan \& Sumathi, 2015 \\
\hline$S$. Typhimurium & $\begin{array}{l}\text { Gong et al., 2010; Patel et al., 2013; Buntin \& Hongpattara- } \\
\text { kere, 2014; Hongpattarakere \& Uraipan, 2014; Peres et al., } \\
\text { 2014; Ren et al., 2014; Jiang et al., 2016; Liu et al., } 2016\end{array}$ \\
\hline Shigella spp. & Venkadesan \& Sumathi, 2015 \\
\hline S. flexneri & $\begin{array}{l}\text { Tambekar \& Bhutada, 2009; Zhu et al., 2014; Davoodabadi } \\
\text { et al., 2015; Liu et al., 2016 }\end{array}$ \\
\hline S. sonnei & $\begin{array}{l}\text { Buntin \& Hongpattarakere, 2014; Davoodabadi et al., 2015; } \\
\text { Pehrson et al., 2015; Liu et al., } 2016\end{array}$ \\
\hline Vibrio parahaemolyticus & Zhu et al., 2014; Jiang et al., 2016 \\
\hline Yersinia enterocolitica & Patel et al., 2013; Davoodabadi et al., 2015 \\
\hline
\end{tabular}


Antimicrobial activity of Lactobacillus plantarum against pathogenic and food spoilage microorganisms

Table 2. Antibacterial activity of Lactobacillus plantarum strains against Gram-positive bacteria

\begin{tabular}{|c|c|}
\hline $\begin{array}{l}\text { Spectrum of } L . \text { plantarum } \\
\text { activity }\end{array}$ & References \\
\hline Bacillus cereus & $\begin{array}{l}\text { Enan et al., 1996; Elegado et al., 2004; Gong et al., 2010; Wang et } \\
\text { al., 2010; Omemu \& Faniran, 2011; Ren et al., 2014; Wen et al., } \\
\text { 2016; Zhang et al., } 2016\end{array}$ \\
\hline B. subtilis & $\begin{array}{l}\text { Elegado et al., 2004; Dal Bello et al., 2007; Valerio et al., 2008; } \\
\text { Gong et al., 2010; Peres et al., 2014; Zhu et al., } 2014\end{array}$ \\
\hline Clostridium difficile & Schoster et al., 2013; Dubourg et al., 2015 \\
\hline C. perfringens & Enan et al., 1996; Gong et al., 2010; Schoster et al., 2013 \\
\hline Enterococcus avium & Inglin et al., 2015 \\
\hline E. casseliflavus & Inglin et al., 2015 \\
\hline E. durans & Inglin et al., 2015 \\
\hline E. faecalis & $\begin{array}{l}\text { Elegado et al., 2004; Todorov \& Dicks, 2005; Dal Bello et al., 2007; } \\
\text { Todorov et al., 2007; Hata et al., 2010; Patel } \text { et al., 2013; Peres et } \\
\text { al., 2014; Todorov et al., 2014; Dubourg et al., 2015; Inglin et al., } \\
2015\end{array}$ \\
\hline E. mundtii & Todorov et al., 2007 \\
\hline Lactobacillus alimentarius & Enan et al., 1996 \\
\hline L. casei & Enan et al., 1996; Elegado et al., 2004 \\
\hline L. curvatus & $\begin{array}{l}\text { Enan et al., 1996; Todorov et al., 2007; Hata et al., 2010; Todorov } \\
\text { et al., 2010; Inglin et al., } 2015\end{array}$ \\
\hline L. delbrueckii & Elegado et al., 2004; Todorov et al., 2010 \\
\hline L. fermentum & Enan et al., 1996; Todorov et al., 2010 \\
\hline L. helveticus & Enan et al., 1996 \\
\hline L. johnsonii & Todorov et al., 2010 \\
\hline L. lindneri & Hata et al., 2010 \\
\hline L. paraplantarum & Todorov et al., 2010 \\
\hline L. pentosus & Enan et al., 1996; Hata et al., 2010; Todorov et al., 2010 \\
\hline L. plantarum & Hata et al., 2010; Elegado et al., 2004 \\
\hline L. rhamnosus & Todorov et al., 2010 \\
\hline L. sakei & Todorov \& Dicks, 2005; Todorov et al., 2007 \\
\hline L. salivarius & Todorov et al., 2007; Todorov et al., 2010 \\
\hline Lactococcus lactis & Enan et al., 1996; Todorov et al., 2007; Todorov et al., 2010 \\
\hline $\begin{array}{l}\text { Leuconostoc } \\
\text { mesenteroides }\end{array}$ & Elegado et al., 2004 \\
\hline Listeria spp. & Dembélé et al., 1998 \\
\hline L. monocytogenes & $\begin{array}{l}\text { Enan et al., 1996; Elegado et al., 2004; Todorov et al., 2007; Gong } \\
\text { et al., 2010; Nielsen et al., 2010; Todorov et al., 2010;Wang et al., } \\
\text { 2010; Rodríguez et al., 2012; Martinez et al., 2013; Buntin \& } \\
\text { Hongpattarakere, 2014; Peres et al., 2014; Todorov et al., 2014; Zhu } \\
\text { et al., 2014; Asurmendi et al., 2015; Dubourg et al., 2015; } \\
\text { Engelhardt et al., 2015; Inglin et al., 2015; Venkadesan \& Sumathi, } \\
\text { 2015; Aoudia et al., 2016; Liu et al., 2016; Wen et al., } 2016\end{array}$ \\
\hline L. ivanovii & Todorov et al., 2010; Inglin et al., 2015 \\
\hline L. grayi & Elegado et al., 2004 \\
\hline
\end{tabular}


Table 2 (cont'd). Antibacterial activity of Lactobacillus plantarum strains against Gram-positive bacteria

\begin{tabular}{|c|c|}
\hline $\begin{array}{l}\text { Spectrum of L. plantarum } \\
\text { activity }\end{array}$ & References \\
\hline Pediococcus sp. & Elegado et al., 2004 \\
\hline P. acidilactici & Elegado et al., 2004 \\
\hline P. pentosaceus & Elegado et al., 2004 \\
\hline Staphylococcus aureus & $\begin{array}{l}\text { Dembélé et al., 1998; Todorov \& Dicks, 2005; Dal Bello et al., } \\
\text { 2007; Gong et al., 2010; Todorov et al., 2010; Wang et al., 2010; } \\
\text { Omemu \& Faniran, 2011; Rodríguez et al., 2012; Patel et al., 2013; } \\
\text { Buntin \& Hongpattarakere, 2014; Peres et al., 2014; Ren et al., } \\
\text { 2014; Zhu et al., 2014; Dubourg et al., 2015; Venkadesan \& Su- } \\
\text { mathi, 2015; Liu et al., } 2016\end{array}$ \\
\hline S. epidermidis & Zhu et al., 2014; Jiang et al., 2016 \\
\hline Streptococcus spp. & Todorov et al., 2007; Todorov et al., 2010 \\
\hline
\end{tabular}

for use as starter cultures in the fermentation of different products and to prolong shelf life (Todorov et al., 2010).

The antibacterial effect of L. plantarum-produced cyclic dipeptides could be explained by the hydrophobic nature of the compounds, which could interfere with outer membrane (Gram-negative) and cytoplasma membrane (Gram-positive) function (Milne et al., 1998; Rhee, 2004). In previous studies, cyclic dipeptides showed a broad spectrum of antibacterial activity (Milne et al., 1998; Ström et al., 2002; Rhee, 2004).

Regarding the inhibition effect caused by LAB and L. plantarum in particular, it is considered that the LAB-produced organic acids, especially lactic and acetic acids, exert a strong inhibitory effect on Gram-negative bacteria (Makras \& De Vuyst, 2006). Some authors observed probiotic-mediated inhibition effect on Escherichia coli and Salmonella Enteritidis that increased proportionally to the concentration of organic acid in the medium. They also stated that low $\mathrm{pH}$ may not be the sole reason for the observed inhibition effects. It could however be an important condition for the passage of organic acids through the membrane to the intracellular environment, where they will accumulate and exert inhibitory activity (Fooks \& Gibson, 2002). Antimicrobial compounds such as phenyllactic acid and lactic acid were effective against many Gram-negative and Gram-positive pathogenic bacteria - Citrobacter freundii, Escherichia coli, Proteus vulgaris, Pseudomonas aeruginosa, Salmonella enterica subsp. enterica, Listeria monocytogenes, Staphylococcus aureus, Bacillus subtilis, Enterococcus faecalis (Dal Bello et al., 2007; Rodríguez et al., 2012).

Because L. plantarum strains are effective against a variety of bacterial pathogens (including methicillin-resistant Staphylococcus aureus and multidrug-resistant enteroaggregative Escherichia coli) they can serve as alternative therapeutic agents against the corresponding infections in humans and animals (Patel et al., 2013; Kumar et al., 2016). For example $L$. plantarum ZDY 2013 can significantly inhibit the adhesion of enterotoxin-producing and pathogenic strains of Bacillus cereus on intestinal epithelial cells by inhibition, competition and displacement (Zhang et al., 2016). According to another 
study, L. plantarum ZDY 2013 pretreatment could play an important role in preventing Helicobacter pylori induced gastric mucosal inflammation and gastric microbiota alteration. These findings suggest that targeting gastric microbiota through oral administration of specific probiotics might be an alternative strategy to prevent $H$. pylori infection (Pan et al., 2016). L. plantarum activity against food spoilage bacteria could be used to prolong shelf life of the food products (Amenu, 2013).

\section{Antifungal activity of L. plantarum}

According to a number of studies L. plantarum has inhibitory activity against many moulds and yeasts, including pathogenic and mycotoxigenic strains from the species Aspergillus, Fusarium, Mucor, Candida, etc. (Table 3). Organic acids are considered one of the main LAB compounds exerting antifungal effects (Russo et al., 2016). Sangmanee \& Hongpattarakere (2014) also found that the antifungal activity of L. plantarum K35 was $\mathrm{pH}$ dependent and favourable to acidic conditions. The major antifungal substances found in that study were lactic acid, 2butyl-4-hexyloctahydro-1H-indene, oleic acid and palmitic acid. On the other hand Niku-Paavola et al. (1999) observed that fungal growth was not inhibited by lactic acid and the active compounds of $L$. plantarum VTT E-78076 were benzoic acid, methylhydantoin, mevalonolactone, cyclo (glycyl-L-leucyl). The major antifungal compounds of L. plantarum MYS6 reported by Deepthi et al. (2016) were 10octadecenoic acid, heptadecanoic acid, methyl ester, palmitic acid, stearic acid and lauric acid. The authors found that these compounds exert inhibitory effect on Fusarium proliferatum growth. In another study L. plantarum 21B showed almost $100 \%$ fungicidal activity against moulds which was due to the phenyllactic and 4-hydroxy-phenyllactic acids (Lavermicocca et al. 2000). There are other experiments that confirm the excellent antifungal activity of phenyllactic acid (Ström et al., 2002; Dal Bello et al., 2007).

According to Ryu et al. (2014) antifungal activity of $L$. plantarum HD1 was due to the 3-hydroxy fatty acids: 5oxododecanoic acid, 3-hydroxy decanoic acid and 3-hydroxy-5-dodecenoic acid.

Some strains of L. plantarum produce cyclic dipeptides with broad spectrum of antifungal activity against moulds and yeasts, such as cyclo(Gly-Leu), cyclo (Phe-Pro), cyclo(Phe-OH-Pro), cyclo (Leu-Pro), which were considered one of the major components responsible for the antifungal activity of these strains (NikuPaavola et al., 1999; Ström et al., 2002; Dal Bello et al., 2007). There are some authors that found different novel peptides obtained from L. plantarum strains exerting antifungal activity due to damage of the cell membrane and consequent leakage of intracellular contents such as $\mathrm{K}^{+}$ ions and ATP (Sharma \& Srivastava, 2014; Muhialdin et al., 2016).

In the study of wide range of potentially useful probiotic strains $L$. plantarum CECT 749 caused $99-100 \%$ aflatoxin reduction in the contaminated bread, promoted by the inhibition of the mycotoxigenic fungi. In that way the bread sample studies showed a shelf life increase of about 3-4 days (Saladino et al., 2016). According to Kurhan \& Cakir (2016) L. plantarum could safely reduce aflatoxin $\mathrm{B}_{1}$ levels without producing any byproducts. Deepthi et al. (2016) reported that the $61.7 \%$ reducing of fumonisin levels by $L$. plantarum MYS6 in their experiment was possibly due to binding mechanism. Some authors found that the 
treatment of wheat seeds with some antimicrobial peptides produced by $L$. plantarum LR/14 prevented fungal growth even after an extended storage under laboratory conditions for around 2.5 years. All fungi examined were inhibited and spore germination was more susceptible than hyphal growth (Gupta \& Srivastava, 2014).

In screening of wide range of 897 LAB isolates for antifungal activity

Table 3. Antifungal activity of Lactobacillus plantarum strains

\begin{tabular}{|c|c|}
\hline $\begin{array}{l}\text { Spectrum of L. plantarum } \\
\text { activity }\end{array}$ & References \\
\hline \multicolumn{2}{|l|}{ Moulds } \\
\hline Aspergillus candidus & Coloretti et al., 2007 \\
\hline A. carbonarius & Djossou et al., 2011 \\
\hline A. flavus & $\begin{array}{l}\text { Lavermicocca et al., 2000; Yang \& Chang, 2010; Ryu et al., } \\
\text { 2014; Sangmanee \& Hongpattarakere, 2014; Muhialdin et al., } \\
\text { 2016; Russo et al., } 2016\end{array}$ \\
\hline A. fumigatus & Ström et al., 2002; Sjögren et al., 2003; Ryu et al., 2014 \\
\hline A. nidulans & Ström et al., 2002; Sjögren et al., 2003; Ryu et al., 2014 \\
\hline A. niger & $\begin{array}{l}\text { Lavermicocca et al., 2000; Dal Bello et al., 2007; Gupta \& } \\
\text { Srivastava, 2014; Yasmin et al., 2015; Russo et al., } 2016\end{array}$ \\
\hline A. ochraceus & Ryu et al., 2014 \\
\hline A. parasiticus & Sangmanee \& Hongpattarakere, 2014; Saladino et al., 2016 \\
\hline A. petrakii & Ryu et al., 2014 \\
\hline A. versicolor & Cheong et al., 2014 \\
\hline Cladosporium spp. & Russo et al., 2016 \\
\hline C. gossypiicola & Ryu et al., 2014 \\
\hline C. herbarum & Cheong et al., 2014 \\
\hline Endomyces fibuliger & Lavermicocca et al., 2000 \\
\hline Eurotium repens & Lavermicocca et al., 2000 \\
\hline E. rubrum & Lavermicocca et al., 2000 \\
\hline Fusarium avenaceum & Niku-Paavola et al., 1999 \\
\hline F. culmorum & Dal Bello et al., 2007; Russo et al., 2016 \\
\hline F. graminearum & Dal Bello et al., 2007 \\
\hline F. oxysporum & Dal Bello et al., 2007 \\
\hline F. proliferatum & Deepthi et al., 2016 \\
\hline F. sporotrichoides & Ström et al., 2002 \\
\hline Mucor spp. & Yasmin et al., 2015 \\
\hline Mucor racemosus & Gupta \& Srivastava, 2014 \\
\hline Monilia sitophila & Lavermicocca et al., 2000 \\
\hline Penicillium spp. & Yasmin et al., 2015 \\
\hline P. chrysogenum & Gupta \& Srivastava, 2014; Russo et al., 2016 \\
\hline P. commune & Ström et al., 2002; Sjögren et al., 2003; Cheong et al., 2014 \\
\hline P. corylophilum & Lavermicocca et al., 2000 \\
\hline P. expansum & Russo et al., 2016; Saladino et al., 2016 \\
\hline P. roqueforti & $\begin{array}{l}\text { Lavermicocca et al., 2000; Sjögren et al., 2003; Ryu et al., 2014; } \\
\text { Muhialdin et al., 2016; Russo et al., } 2016\end{array}$ \\
\hline P. solitum & Cheong et al., 2014 \\
\hline Rhizopus stolonifer & Gupta \& Srivastava, 2014 \\
\hline
\end{tabular}


Antimicrobial activity of Lactobacillus plantarum against pathogenic and food spoilage microorganisms

Table 3 (cont'd). Antifungal activity of Lactobacillus plantarum strains

\begin{tabular}{ll}
\hline $\begin{array}{l}\text { Spectrum of L. plantarum } \\
\text { activity }\end{array}$ & References \\
\hline Yeasts & \\
Candida albicans & Ström et al., 2002; Wynne et al., 2004; Ryu et al., 2014; Sharma \\
& \& Srivastava, 2014 \\
Debaryomyces hansenii & Ström et al., 2002 \\
Kazachstania exigua & Ryu et al., 2014 \\
Kluyveromyces marxianus & Ström et al., 2002; Sjögren et al., 2003 \\
Pichia anomala & Ström et al., 2002; Sjögren et al., 2003 \\
$\begin{array}{l}\text { P. kudriavzevii } \\
\text { Saccharomyces bulderi }\end{array}$ & Ryu et al., 2014 \\
S. cerevisiae & Ryu et al., 2014 \\
S. servazzii & Ström et al., 2002; Jiang et al., 2016 \\
Rhodotorula mucilaginosa & Ryu et al., 2014 \\
& Ström et al., 2002; Sjögren et al., 2003; Inglin et al., 2015; \\
& Jiang et al., 2016 \\
\hline
\end{tabular}

against cheese spoilage moulds was found that 12 isolates possessed strong antifungal activity, and all of them were identified as L. plantarum. Further studies indicated that all L. plantarum isolates prevented the visible growth of Penicillium commune FRR 4117 on cottage cheese by 14-25 days longer than cottage cheese without added LAB with antifungal activity (Cheong et al., 2014).

L. plantarum LR/14 showed potent fungicidal activity against Candida albicans SC5314 by affecting cell viability, membrane permeability and biofilm formation, thereby the authors suggested this strain as a probable natural candidate therapeutic agent (Sharma \& Srivastava, 2014). Some authors reported powerful inhibitory activity of tetracycline-resistant L. plantarum strain against Candida albicans that may make this probiotic useful for improved management of yeast-related conditions such as thrush and irritable bowel syndrome (Wynne et al., 2004). Also, administration of $L$. plantarum is sometimes helpful for reducing the clinical symptoms and prevention of fungal infections (De Seta et al., 2014).

\section{CONCLUSIONS}

Based on a wide range of investigations, L. plantarum should be considered an important LAB species with excellent antimicrobial activity. Because of that some L. plantarum strains can be used as effective biopreservatives in food industry or supporting therapeutic agents in treatment of infections caused by susceptible microorganisms. Antimicrobial compounds produced by $L$. plantarum such as bacteriocins and organic acids, could also be applied as alternatives of preservatives and therapeutics.

\section{REFERENCES}

Abo-Amer, A. E., 2013. Inhibition of foodborne pathogens by a bacteriocin-like substance produced by a novel strain of $\mathrm{Lac}$ tobacillus acidophilus isolated from camel milk. Applied Biochemistry and Microbiology, 49, 270-279.

Alakomi, A. L., E. Skytta, M. Saarela, T. Mattila-Sandholm, K. Latva-Kala \& L. M. Helander, 2000. Lactic acid permeabilizes Gram-negative bacteria by disrupting the 
outer membrane. Applied and Environmental Microbiology, 66, 2001-2005.

Amenu, D., 2013. Antimicrobial activity of lactic acid bacteria isolated from "Ergo", Ethiopian traditional fermented milk. Current Research in Microbiology and Biotechnology, 1, 278-284.

Aoudia, N., A. Rieu, R. Briandet, J. Deschamps, J. Chluba, G. Jego, C. Garrido \& J. Guzzo, 2016. Biofilms of Lactobacillus plantarum and Lactobacillus fermentum: Effect on stress responses, antagonistic effects on pathogen growth and immunomodulatory properties. Food Microbiology, 53, 51-59.

Arasu, M., N. Al-Dhabi, S. Ilavenil, K. Choi \& S. Srigopalram, 2016. In vitro importance of probiotic Lactobacillus plantarum related to medical field. Saudi Journal of Biological Sciences, 23, 6-10.

Arias, O., M. de la Luz Reyes, V. Navarro, C. Solis, G. Marquez, S. Sanchez, C. Snell \& R. Zuniga, 2013. Antagonistic effect of probiotic strains against two pathogens: Salmonella typhimurium and E. coli O157:H7 resistant to antibiotics. e-Gnosis, 11, 1-16.

Asurmendi, P., M. Garćia, L. Pascual. \& L. Barberis, 2015. Biocontrol of Listeria monocytogenes by lactic acid bacteria isolated from brewer's grains used as feedstuff in Argentina. Journal of Stored Products Research, 61, 27-31.

Broberg, A., K. Jacobsson, K. Ström \& J. Schnürer, 2007. Metabolite profiles of lactic acid bacteria in grass silage. Applied and Environmental Microbiology, 73, 5547-5552.

Buntin, N. \& T. Hongpattarakere, 2014. Antimicrobial activity and plantaricin (pln) encoding genes of Lactobacillus plantarum isolated from various sources. Journal of Biotechnology (Abstracts), 185, 74.

Byczkowski, J. \& T. Gessner, 1988. Biological role of superoxide ion-radical. International Journal of Biochemistry, 20, 569580 .

BJVM, 21, No 3
Cebeci, A. \& C. Gürakan, 2003. Properties of potential probiotic Lactobacillus plantarum strains. Food Microbiology, 20, 511518.

Cheong, E., A. Sandhu, J. Jayabalan, T. Le, N. Nhiep, H. Ho, J. Zwielehner, N. Bansal \& M. Turner, 2014. Isolation of lactic acid bacteria with antifungal activity against the common cheese spoilage mould Penicillium commune and their potential as biopreservatives in cheese. Food Control, 46, 91-97.

Coloretti, F., S. Carri, E. Armaforte, C. Chiavari, L. Grazia \& C. Zambonelli, 2007. Antifungal activity of lactobacilli isolated from salami. FEMS Microbiology Letters, 271, 245-250.

Dal Bello, F., C. Clarke, L. Ryan, H. Ulmer, T. Schober, K. Ström, J. Sjögren, D. van Sinderen, J. Schnürer \& E. Arendt, 2007. Improvement of the quality and shelf-life of wheat bread by fermentation with the antifungal strain Lactobacillus plantarum FST 1.7. Journal of Cereal Science, 45, 309-318.

Dalié, D., A. Deschamps \& F. Richard-Forget, 2010. Lactic acid bacteria - potential for control of mould growth and mycotoxins: A Review. Food Control, 21, 370-380.

Davoodabadi, A., M. Dallal, A. Foroushani, M. Douraghi, M. Yazdi \& F. Harati, 2015. Antibacterial activity of Lactobacillus spp. isolated from feces of healthy infants against enteropathogenic bacteria. Anaerobe, 34, 53-58.

De Seta, F., F. Parazzini, R. De Leo, R. Banco, G. Maso, D. De Santo, A. Sartore, G. Stabile, S. Inglese, M. Tonon \& S. Restaino, 2014. Lactobacillus plantarum P17630 for preventing Candida vaginitis recurrence: A retrospective comparative study. European Journal of Obstetrics, Gynecology and Reproductive Biology, 182, 136-139.

Deepthi B. V., K. Poornachandra Rao, G. Chennapa, M. K. Naik, K. T. Chandrashekara \& M. Y. Sreenivasa, 2016. Antifungal attributes of Lactobacillus planta- 
rum MYS6 against fumonisin producing Fusarium proliferatum associated with poultry feeds. PLoS One, 11, https://dx.doi.org/10.1371/journal.pone. 0155122 (in press).

Dembélé, T., V. Obdržálek \& M. Votava, 1998. Inhibition of bacterial pathogens by Lactobacilli. Zentralblatt für Bacteriologie, 288, 395-401.

Denev, S., G. Beev, T. Dinev, R. Moutafchieva \& T. Georgieva, 2015. Health benefits of functional probiotic cultures. In: Scientific Symposium Beneficial and Pathogenic Microbes for Healthier Life and Safety Foods (Abstracts), Sofia, Bulgaria, United Scientific Group, Sofia, p. 1.

Djossou, O., I. Perraud-Gaime, F. Mirleau, G. Rodriguez-Serrano, G. Karou, S. Niamke, I. Ouzari, A. Boudabous \& S. Roussos, 2011. Robusta coffee beans post-harvest microflora: Lactobacillus plantarum sp. as potential antagonist of Aspergillus carbonarius. Anaerobe, 17, 267-272.

Dubourg, G., Z. Elsawi \& D. Raoult, 2015. Assessment of the in vitro antimicrobial activity of Lactobacillus species for identifying new potential antibiotics. International Journal of Antimicrobial Agents, 46, 590-593.

Earnshaw, R., 1992. The antimicrobial action of lactic acid bacteria: Natural food preservation system. In: The lactic Acid Bacteria in Health and Disease, ed. B. Wood, Elsevier Applied Science, London and New York, pp. 211-232.

Eklund, T., 1984. The effect of carbondioxide on bacterial growth and on uptake processes in the bacterial membrane vesicles. International Journal of Food Microbiology, 1, 179-185.

Elegado, F., M. Guerra, R. Macayan, H. Mendoza \& M. Lirazan, 2004. Spectrum of bacteriocin activity of Lactobacillus plantarum BS and fingerprinting by RAPDPCR. International Journal of Food Microbiology, 95, 11-18.

Enan, G., A. El-Essawy, M. Uyttendaele \& J. Debevere, 1996. Antibacterial activity of
Lactobacillus plantarum isolated from dry sausage: characterization production and bactericidal action of plantaricin UG1. International Journal of Food Microbiology, 30, 189-215.

Engelhardt, T., H. Albano, G. Kiskó, C. Mohácsi-Farkas \& P. Teixeira, 2015. Antilisterial activity of bacteriocinogenic Pediococcus acidilactici HA6111-2 and Lactobacillus plantarum ESB 202 grown under $\mathrm{pH}$ and osmotic stress conditions. Food Microbiology, 48, 109-115.

Fooks, L. \& G. Gibson, 2002. Probiotics as modulators of the gut flora. British Journal of Nutrition, 88, Suppl 1, 39-49.

Gerez, C., M. Torres, G. de Valdez \& G. Rollán, 2013. Control of spoilage fungi by lactic acid bacteria. Biological Control, 64, 231-237.

Gong, H., X. Meng \& H. Wang, 2010. Plantaricin MG active against Gram-negative bacteria produced by Lactobacillus plantarum KLDS1.0391 isolated from "Jiaoke", a traditional fermented cream from China. Food Control, 21, 89-96.

Gupta, R. \& S. Srivastava, 2014. Antifungal effect of antimicrobial peptides (AMPs LR14) derived from Lactobacillus plantarum strain LR/14 and their applications in prevention of grain spoilage. Food Microbiology, 42, 1-7.

Haller, D., H. Colbus, M. Gänzle, P. Scherenbacher, C. Bode \& W. Hammes, 2001. Metabolic and functional properties of lactic acid bacteria in the gastro-intestinal ecosystem: a comparative in vitro study between bacteria of intestinal and fermented food origin. Systematic and Applied Microbiology, 24, 218-226.

Hata, T., R. Tanaka \& S. Ohmomo, 2010. Isolation and characterization of plantaricin ASM1: A new bacteriocin produced by Lactobacillus plantarum A-1. International Journal of Food Microbiology, 137, 94-99.

Hongpattarakere, T. \& S. Uraipan, 2014. Antagonistic activity of Lactobacillus plantarum and saba banana starch under proxi- 
mal colon model challenged with Salmonella typhimurium. Journal of Biotechnology (Abstracts), 185, 76.

Inglin, R., M. Stevens, L. Meile, C. Lacroax \& L. Meile, 2015. High-throughput screening assays for antibacterial and antifungal activities of Lactobacillus species. Journal of Microbiological Methods, 114, 26-29.

Jiang, H., P. Li \& Q. Gu, 2016. Heterologous expression and purification of plantaricin NC8, a two-peptide bacteriocin against Salmonella spp. from Lactobacillus plantarum ZJ316. Protein Expression and Purification, 127, 28-34.

Khan, I. \& S. Kang, 2016. Probiotic potential of nutritionally improved Lactobacillus plantarum DGK-17 isolated from Kimchi - a traditional Korean fermented food. Food Control, 60, 88-94.

Kleerebezem, M., J. Boekhorst, R. van Kranenburg, D. Molenaar, O. Kuipers, R. Leer, R. Tarchini, S. Peters, H. Sandbrink, M. Fiers, W. Stiekema, R. Lankhorst, P. Bron, S. Hoffer, M. Groot, R. Kerkhoven, M. de Vries, B. Ursing, W. de Vos \& R. Siezen, 2003. Complete genome sequence of Lactobacillus plantarum WCFS1. PNAS, 100, 1990-1995.

Kumar, M., P. Dhaka, D. Vijay, J. Vergis, V. Mohan, A. Kumar, N. Kurkure, S. Barbuddhe, S. Malik \& D. Rawool, 2016. Antimicrobial effects of Lactobacillus plantarum and Lactobacillus acidophilus against multidrug-resistant enteroaggregative Escherichia coli. International Journal of Antimicrobial Agents, 48, 265-270.

Kurhan, Ş. \& I. Çakir, 2016. DNA-bioprotective effects of lactic acid bacteria against aflatoxin $\mathrm{B}_{1}$. Current Research in Nutrition and Food Science, 4, SI 2, 87-91.

Lavermicocca, P., F. Valerio, A. Evidente, S. Lazzaroni, A. Corsetti \& M. Cobbetti, 2000. Purification and characterization of novel antifungal compounds from the sourdough Lactobacillus plantarum strain 21B. Applied and Environmental Microbiology, 66, 4084-4090.
Liu, H., L. Zhang, H. Yi, X. Han \& C. Chi, 2016. Identification and characterization of plantaricin Q7, a novel plantaricin produced by Lactobacillus plantarum Q7. LWT - Food Science and Technology, 71, 386-390.

Magnusson, J., K. Ström, S. Roos, J. Sjögren \& J. Schnürer, 2003. Broad and complex antifungal activity among environmental isolates of lactic acid bacteria. FEMS Microbiology Letters, 219, 129-135.

Markas, L. \& L. De Vuyst, 2006. The in vitro inhibition of Gram-negative pathogenic bacteria by bifidobacteria is caused by the production of organic acids. International Dairy Journal, 16, 1049-1057.

Martinez, R., M. Wachsman, N. Torres, J. LeBlanc, S. Todorov \& B. de Melo Franco, 2013. Biochemical, antimicrobial and molecular characterization of a noncytotoxic bacteriocin produced by Lactobacillus plantarum ST71KS. Food Microbiology, 34, 376-381.

Milne, P., A. Hunt, K. Rostoll, J. van der Walt \& C. Graz, 1998. Medicinal chemistry: The biological activity of selected cyclic dipeptides. Journal of Pharmacy and Pharmacology, 50, 1331-1337.

Muhialdin, B., Z. Hassan, F. Bakar \& N. Saari, 2016. Identification of antifungal peptides produced by Lactobacillus plantarum IS10 grown in the MRS broth. Food Control, 59, 27-30.

Nielsen, D., G.-S. Cho, A. Hanak, M. Huch, C. Franz \& N. Arneborg, 2010. The effect of bacteriocin-producing Lactobacillus plantarum strains on the intracellular $\mathrm{pH}$ of sessile and planktonic Listeria monocytogenes single cells. International Journal of Food Microbiology, 141, 53-59.

Niku-Paavola, M.-L., A. Laitila, T. MattilaSandholm \& A. Haikara, 1999. New types of antimicrobial compounds produced by Lactobacillus plantarum. Journal of Applied Microbiology, 86, 29-35.

Omemu, A. \& O. Faniran, 2011. Assessment of the antimicrobial activity of lactic acid bacteria isolated from two fermented 
maize products - ogi and kunni-zaki. $M a$ laysian Journal of Microbiology, 7, 124 128.

O'Sullivan, L., R. Ross \& C. Hill, 2002. Potential of bacteriocin-producing lactic acid bacteria for improvements in food safety and quality. Biochimie, 84, 593-604.

Pan, M., C. Wan, Q. Xie, R. Huang, X. Tao, N. P. Shah \& H. Wei, 2016. Changes in gastric microbiota induced by Helicobacter pylori infection and preventive effects of Lactobacillus plantarum ZDY 2013 against such infection. Journal of Dairy Science, 99, 970-981.

Patel, A., N. Shah, P. Ambalam, J. Prajapati, O. Holst \& A. Ljungh, 2013. Intimicrobial profile of lactic acid bacteria isolated from vegetables and indigenous fermented foods of India against clinical pathogens using microdilution method. Biomedical and Environmental Sciences, 26, 759-764.

Pehrson, M. E. S. F., I. M. Mancilha \& C. A. S. Pereira, 2015. Antimicrobial activity of probiotic Lactobacillus strains towards gram-negative enteropathogens. European International Journal of Science and Technology, 4, 136-149.

Peres, C., M. Alves, A. Hernandez-Mendoza, L. Moreira, S. Silva, M. Bronze, L. VilasBoas, C. Peres \& F. Malcata, 2014. Novel isolates of lactobacilli from fermented Portuguese olive as potential probiotics. $L W T$ - Food Science and Technology, 59, 234246.

Perez, R.H., T. Zendo \& K. Sonomoto, 2014. Novel bacteriocins from lactic acid bacteria (LAB): various structures and applications. Microbial Cell Factories, 13, Suppl $1,1-13$.

Ren, D., C. Li, Y. Qin, R. Yin, S. Du, F. Ye, C. Liu, H. Liu, M. Wang, Y. Li, Y. Sun, X. Li, M. Tian \& N. Jin, 2014. In vitro evaluation of the probiotic and functional potential of Lactobacillus strains isolated from fermented food and human intestine. Anaerobe, 30, 1-10.

Rhee, K.-H., 2004. Cyclic dipeptides exhibit synergistic, broad spectrum antimicrobial effects and have anti-mutagenic properties. International Journal of Antimicrobial Agents, 24, 423-427.

Rodríguez, N., J. Salgado, R. RodríguezSolana, S. Cortés \& J. Domínguez, 2012. Biotechnological production of cell-free extract obtained by fermentation of cheese whey and phenylpyruvic acid using Lactobacillus plantarum and antimicrobial effect against pathogen bacteria. New Biotechnology, 29, 59.

Russo, P., M. Arena, D. Fiocco, V. Capozzi, D. Drider \& G. Spano, 2016. Lactobacillus plantarum with broad antifungal activity: A promising approach to increase safety and shelf-life of cereal-based products. International Journal of Food Microbiology, http://dx.doi.org/10.1016/j. ijfoodmicro.2016.04.027 (in press).

Ryu, E., E. Yang, E. Woo \& H. Chang, 2014. Purification and characterization of antifungal compounds from Lactobacillus plantarum HD1 isolated from kimchi. Food Microbiology, 41, 19-26.

Saladino, F., C. Luz, L. Manyes, M. Fernández-Franzón \& G. Meca, 2016. In vitro antifungal activity of lactic acid bacteria against mycotoxigenic fungi and their application in loaf bread shelf life improvement. Food Control, 67, 273-277.

Sangmanee, P. \& T. Hongpattarakere, 2014. Inhibitory of multiple antifungal components produced by Lactobacillus plantarum $\mathrm{K} 35$ on growth, aflatoxin production and ultrastructure alterations of Aspergillus flavus and Aspergillus parasiticus. Food Control, 40, 224-233.

Schoster, A., B. Kokotovic, A. Permin, P. Pedersen, F. Dal Bello \& L. Guardabassi, 2013. In vitro inhibition of Clostridium difficile and Clostridium perfringens by commercial probiotic strains. Anaerobe, 20, 36-41.

Sharma, A. \& S. Srivastava, 2014. AntiCandida activity of spent culture filtrate of Lactobacillus plantarum strain LR/14. Journal de Mycologie Médicale, 24, 2534. 
Sjögren, J., J. Magnusson, A. Broberg, J. Schnürer \& L. Kenne, 2003. Antifungal 3hydroxy fatty acids from Lactobacillus plantarum MiLAB 14. Applied and Environmental Microbiology, 69, 7554-7557.

Ström, K., J. Sjögren, A. Broberg \& J. Schnürer, 2002. Lactobacillus plantarum MiLAB 393 produces the antifungal cyclic dipeptides cyclo(L-Phe-L-Pro) and cyclo(L-Phe-trans-4-OH-L-Pro) and 3phenyllactic acid. Applied and Environmental Microbiology, 68, 4322-4327.

Sunanliganon C., D. Thong-Ngam, S. Tumwasorn \& N. Klaikeaw, 2012. Lactobacillus plantarum B7 inhibits Helicobacter pylori growth and attenuates gastric inflammation. World Journal of Gastroenterology, 18, 2472-2480.

Tambekar, D. \& S. Bhutada, 2009. Studies on antimicrobial activity and characteristics of bacteriocins produced by Lactobacillus strains isolated from milk of domestic animals. The Internet Journal of Microbiology, 8, 1-5.

Todorov, S. \& L. Dicks, 2005. Lactobacillus plantarum isolated from molasses produced bacteriocins active against Gramnegative bacteria. Enzyme and Microbial Technology, 36, 318-326.

Todorov, S., H. Nyati, M. Meincken \& L. Dicks, 2007. Partial characterization of bacteriocin AMA-K, produced by Lactobacillus plantarum AMA-K isolated from naturally fermented milk from Zimbabwe. Food Control, 18, 656-664.

Todorov, S., M. Wachsman, T. IgnatovaIvanova \& I. Ivanova, 2014. Lactobacillus plantarum ST16Pa - are we ready to use it as bio-protective culture? Bulgarian Journal of Agricultural Science, 20, Suppl 1, 55-58.

Todorov, S., P. Ho, M. Vaz-Velho \& L. Dicks, 2010. Characterization of bacteriocins produced by two strains of Lactobacillus plantarum isolated from Beloura and Chouriço, traditional pork products from Portugal. Meat Science, 84, 334-343.
Urga, K., B. Gashe, A. Fite \& A. Nigatu, 1992. Changes in acidity and lactic acid production during Ititu fermentation. Ethiopian Journal of Agricultural Science, 9, 91-95.

Valerio, F., P. De Bellis, S. Lonigro, A. Visconti \& P. Lavermicocca, 2008. Use of Lactobacillus plantarum fermentation products in bread-making to prevent Bacillus subtilis ropy spoilage. International Journal of Food Microbiology, 122, 328332.

Venkadesan, D. \& V. Sumathi, 2015. Screening of lactic acid bacteria for their antibacterial activity against milk borne pathogens. International Journal of Applied Research, 1, 970-973.

Wang, C.-Y., P.-R. Lin, C.-C. Ng \& Y.-T Shyu, 2010. Probiotic properties of Lactobacillus strains isolated from the feces of breast-fed infants and Taiwanese pickled cabbage. Anaerobe, 16, 578-585.

Wen, L., K. Philip \& N. Ajam, 2016. Purification, characterization and mode of action of plantaricin K25 produced by Lactobacillus plantarum. Food Control, 60, 430 439.

Woo, J.Y., W. Gu, K.A. Kim, S.E. Jang, M.J. Han \& D.H. Kim, 2014. Lactobacillus pentosus var. plantarum C29 ameliorates memory impairment and inflammaging in a D-galactose-induced accelerated aging mouse model. Anaerobe, 27, 22-26.

Wynne, A., A. McCartney, J. Brostoff, B. Hudspith \& G. Gibson, 2004. An in vitro assessment of the effects of broadspectrum antibiotics on the human gut microflora and concomitant isolation of a Lactobacillus plantarum with antiCandida activities. Anaerobe, 10, 165169.

Yang, E. \& H. Chang, 2010. Purification of a new antifungal compound produced by Lactobacillus plantarum AF1 isolated from kimchi. International Journal of Food Microbiology, 139, 56-63.

Yasmin, A., M. Ashraf, M. Arshad, G. Muhammad, M. Zahid \& B. Mustafa, 2015. Determination of biopreservative effects of 
Antimicrobial activity of Lactobacillus plantarum against pathogenic and food spoilage microorganisms

bacteriocins isolated from lactic acid producing bacteria against food spoiling fungi. International Journal of Current Microbiology and Applied Sciences, 4, 88-96.

Zhang, Z., X. Tao, N. P. Shah \& H. Wei, 2016. Antagonistics against pathogenic Bacillus cereus in milk fermentation by Lactobacillus plantarum ZDY2013 and its anti-adhesion effect on Caco-2 cells against pathogens. Journal of Dairy Science, 99, 2666-2674.

Zhu, X., Y. Zhao, Y. Sun \& Q. Gu, 2014. Purification and characterization of plantaricin ZJ008, a novel bacteriocin against Staphylococcus spp. from Lactobacillus plantarum ZJ008. Food Chemistry, 165 216-223.

Paper received 09.11.2016; accepted for publication 06.02.2017

\section{Correspondence:}

Assis. Prof. Toncho Dinev Faculty of Agriculture, Trakia University, 6000 Stara Zagora, Bulgaria, e-mail:dinev_sz@mail.bg 